\title{
Performance of a Brazilian population sample in the Boston Diagnostic Aphasia Examination. A pilot study
}

M. Radanovic ${ }^{1}$ and L.L. Mansur ${ }^{1,2}$

\author{
Departamentos de ${ }^{1} \mathrm{~N}$ eurologia and ${ }^{2}$ Fisioterapia, Fonoaudiologia e Terapia \\ O cupacional, Faculdade de Medicina, Universidade de São Paulo, São Paulo, \\ SP, Brasil
}

\begin{abstract}
\section{Correspondence}

M. Radanovic

R. Cristiano Viana, 163/92

05411-000 São Paulo, SP

Brasil

Fax: + 55-11-3088-9708

E-mail: radano@ tecway.com.br

Publication supported by FAPESP.

Received July 17, 2001

Accepted December 11, 2001

Brazilian researchers and health professionals often face the challenge of having to use tests developed in foreign languages and standardized for populations of other countries, especially in the fields of Neuropsychology and Neurolinguistics. This fact promotes a feeling that some scoring systems may be inadequate for our sociocultural reality. In the present study, we describe the performance of a Brazilian population sample submitted to a translated and adapted version of the Boston Diagnostic Aphasia Examination (BDAE). Sixty normal volunteers (21 men and 39 women), all Portuguese native speakers, ranging in age from 15 to 78 years (average 43.7) and with an educational level of 2 to 16 years (average 9.9), were tested using a translated and adapted Portuguese version of the BDAE. Cut-off scores are suggested for our population and the performance of the Brazilian sample is compared to that of American and Colombian samples, with the results being closely similar in all tasks. We also performed a correlation analysis between age, gender and educational level and the influence of these variables on the performance of the subjects. We found no statistically significant differences between genders. Educational level correlated positively with performance, especially in the subtests involving reading and writing. There was a negative correlation between age and performance in two subtests (Visual Confrontation Naming and Sentences to Dictation), but a coexisting effect of educational level could not be ruled out.
\end{abstract}

\author{
Key words \\ - Aphasia \\ - Diagnosis \\ - Portuguese
}

\section{Introduction}

One of the great difficulties faced by Brazilian health professionals is the absence of diagnostic tools standardized in Portuguese and adapted to their sociocultural reality. This is particularly true in the area of Neurolinguistics and in others related to Neuropsychology, since professionals and researchers use imported diagnostic resources from other cultures (generally Anglo-Saxon) translated into Portuguese. This creates a series of difficulties in interpreting the data. These difficulties are related to the very structure of the language (Portuguese versus English) and its peculiarities, as well as the cultural context of each country. Thus, the researcher faces the task of not only "translating" but also adapting the diagnostic tool equally, in an attempt to minimize the mar- 
gin of interpretive error. Besides, for the most part the performance of the local normal population to the test in question is unknown. Foreign cut-off scores are used, increasing the distortion of the conclusions. Furthermore, the use of language is considerably influenced by gender, age and educational level.

The main questions we propose to discuss in this paper are:

- What is the standard performance of the Brazilian population in the Boston Diagnostic Aphasia Examination (BDAE)?

- Does the standard differ substantially from the performance of other populations where this test is the norm?

- What is the impact of low education (very common in the public we usually attend) on the scores of the various subtests?

- What adaptations should be made to make the test more compatible with our reality?

The BDAE was developed by Goodglass and Kaplan in 1972 (1) and reviewed in 1983 (2). These investigators suggest its application to detect and classify aphasic syndromes, determine their severity (transversely and longitudinally) and to supply indicators for language therapy. When the initial and more recent versions are compared, the main modifications in the most recent one are the inclusion of the Body-Part Identification subtest, the stimulus re-ordination, the method for recording and computing data and the statistical analysis (considering percentiles instead of Z-scores). This new version also includes subcortical aphasia analysis based on recent views about the role of subcortical structures in language. In the Spanish adaptation $(3,4)$, criteria closely similar to those used in the Portuguese adaptation were applied: stimuli with strong cultural connotations (texts for comprehension) and those which failed to fulfill the aims of the task after translation (due to syllabic and phonemic constitution changes) were replaced. In addition, the characteristics of some visual stimuli were modified.

The development of neuroimaging made the use of BDAE less important to infer lesion sites (as initially proposed), but did not invalidate its potential to establish a common language among the several areas involved in aphasia evaluation. In other words, the BDAE can reflect the view of those interested in neuropathological correlations as much as psycholinguistic analysis.

Although conceived a number of decades ago, the BDAE still maintains many currently valid characteristics. This fact particularly applies to the semantic treatment of items by categories proposed in the test. Goodglass et al. (5), observing 72 fluent and non-fluent aphasics, pointed out differences in the performance of recognizing and naming the different semantic categories of the BDAE. Fluent aphasics presented a better performance in letter naming, but had great difficulty in object naming, whereas the inverse was detected in non-fluent aphasics. The categories relative to colors, shapes and actions all contributed less notably to differentiating subgroups. At that time the author considered the possibility of the lexical access system being anatomically subdivided according to the "psychological" characteristics of the different categories and also according to the sensory input modality through which naming is "constructed".

The current debate about the semantic system is ample and includes many topics not yet answered. How to consider specific loss of categories? This question is widely debated by defenders of the idea of a single semantic system based on a connectionist network model and by defenders of its organization on a sensory modality basis $(6,7)$. How to consider the dissociation between loss of knowledge related to verbs and nouns? This theme induces reflections about syntactic and semantic representations linked to these categories (8). From the neuroanatomic point of view, with the advent of modern study techniques, this subject has been fre- 
quently revisited in publications (9-12) that present ever more data to answer the complex questions about the neural substrate supporting the thinking process.

In the still emergent field of Neurolinguistics, possibly the strongest arguments for the use of the BDAE are its sensitivity in detecting aphasia (being a "scanning" test) and the possibility of comparison with studies performed in many countries, given that the test is widely accepted and frequently cited in the literature (13).

The test was standardized in an American population of 147 subjects ranging in age from 25 to 85 years and with an educational level ranging from less than 9 years through college. Normative studies for Spanish-speaking people have also been published $(3,4)$. The test seeks to evaluate language aspects that can be altered due to left hemispheric lesions, namely articulation, fluency, word-finding difficulty, repetition, automatized speech, loss of grammar and syntax, paraphasias, auditory comprehension, reading, and writing.

\section{Procedures, Population and Methods}

\section{Test characteristics}

The BDAE examines comprehension and production procedures in oral and written modalities. Most of the subtests consider the stimuli within deliberately decontextualized situations. Stimuli are presented through auditory or visual input in order to assure the analysis of oral and written comprehension. The tasks require greater or lesser emphasis on automatic information or semantic processing. Cards are used for the purpose of narration or to represent concepts of six semantic categories, phrases of different degrees of probability and length and texts that differ in complexity and length for interpretation. Semantic categories include nouns related to objects, shapes, numbers, letters and colors. The test considers not only nouns but also transitive and intransitive action verbs. One last category includes stimuli related to parts of the body.

Although all language production subtests lend themselves to the evaluation of pathologies frequently associated with aphasia, such as apraxia of speech and dysarthria, some are more specific, involving tasks such as movements of phonoarticulatory organs and the repetition of words which seek control of emission time for items with different phonological density and syllabic extension. The stimuli are presented in such a way as to permit the differentiation of linguistic and nonlinguistic factors from perception to the integration of elements which constitute the meaning. The recognition of geometric shapes, letters and syllables is required, as well as the recognition and interpretation of words, phrases and texts. Some subtests are concerned with residual abilities regarding their potential role in language therapy. This is the case for recitation, singing and rhythm, which are skills presumably linked to the "non-dominant" hemisphere.

\section{Scoring}

All subtests are scored. The production subtests (which are included in the categories Oral Agility, Repetition, Naming and Oral Reading) are scored in a more rigorous way, considering qualitative data, answer delay and sensitivity to phonemic or semantic clues. Other qualitative data relative to language production (articulation, praxis, semantic and phonemic exchanges) are computed throughout the evaluation in such a manner that it is possible to discriminate the influence of variables such as programming and articulatory difficulties on linguistic performance. Partial answers are also considered.

From a qualitative point of view, it is possible to determine the influence of input and output type. Naming can be verified in the subtest using visual confrontation or au- 
ditory presentation of the semantic characteristics of the target stimuli. The output can be written or oral. The same stimuli presented for recognition are also used to verify the preservation of their representation in the semantic system.

\section{Portuguese translation and adaptation}

The Portuguese version and adaptation of the BDAE (Mansur LL and Mor L, unpublished results) were based on the most current original English version (2). The process primarily took into account the aims of each subtest, looking for similar resources in Portuguese, which made the simple translation of all stimuli impossible. We will briefly present the criteria used for translating and/ or adaptating the subtests.

Translation. The following subtests were composed using the original English stimuli, where only translation into Portuguese was used: Word Discrimination (the items presented on the cards used in this task were selected according to the frequency of occurrence in English and there is no similar linguistic study in Brazil), Body-Part Identification, Commands, Nonverbal Agility, Automatized Sequences, Oral Reading (words), Responsive Naming, Visual Confrontation Naming, Animal Fluency, WordPicture Matching, Serial Writing (although the absence of $\mathrm{K}, \mathrm{W}$ and $\mathrm{Y}$ is not considered as an error), Written Confrontation Naming, Narrative Writing and Sentences to Dictation.

Translation and adaptation. The following subtests had to be adapted in order to assure that the purpose of the task was maintained:

- Complex Ideational Material: the sentences used for the semantic judgment task and the canonical narratives were only translated. Three of the texts were adapted, with attributes of proposition organization, lexical complexity, number of explicit inferences and macrostructure of the original text being maintained. Texts demanding "world knowledge" distinct from Brazilian reality (as in the "trumpeter in the quarters" and "a fire in the hotel", both humorous and with greater implicit information) were replaced, keeping the characteristics of humor and using popular stories.

- Verbal Agility: stimuli were adapted to maintain syllabic extension and phonological density similar to the original.

- Reciting and Singing: stimuli were replaced with Brazilian popular songs and stories.

- Repetition of Words: the expression "Episcopal Methodist" was replaced with "Apostolic Catholic" (católico apostólico), considered more suitable to the frequency of the phrase in Portuguese.

- Repetition of Phrases: the phrases were replaced, keeping the "predictability effect" and its possible facilitation role in lexical production. Attributes such as coordinated or subordinated constructions, the presence of lexicon or grammatical words and lexical complexity were respected.

- Oral Sentence Reading: the same stimuli adapted to the repetition task were used, as in the English version.

- Symbol Discrimination: translation would invalidate the monosyllabic quality of stimuli, so they were replaced.

- Word Recognition: the target word, with two syllables, should be inserted into an option set related in terms of phonological and semantic criteria as well as aleatory distractors.

- Comprehension of Oral Spelling: stimuli were changed respecting the necessity for an increasing length and different degrees of irregularities in the phoneme-grapheme conversion.

- Sentence and Paragraph Reading Comprehension: those paragraphs in which content was related to the American reality (such as the texts about "work relations" and "government responsibilities") were replaced taking into account the paragraph extension, 
syntactic and lexical complexity, in addition to the degree of inferences involved in the comprehension.

- Primer-Level Dictation: the item sequence according to increasing complexity was maintained: letters, numbers and words (mono- or disyllabic) of high frequency and with strong phoneme-grapheme correspondence.

- Spelling to Dictation: this task was composed of disyllabic, trisyllabic or polysyllabic words with a greater number of irregularities.

\section{Brazilian sample}

The Portuguese version of the BDAE was applied to 60 Brazilian volunteers (39 women and $21 \mathrm{men}$ ), who were healthy, independent for daily life activities, without neurological or psychiatric antecedents and all Portuguese native speakers living in São Paulo for more than 10 years. Subjects were recruited among speech pathology students' relatives, hospital employees and outpatients' relatives or caregivers, aleatorily, but trying to form subgroups which could represent preliminary references regarding gender, age and schooling influences on the performance of normal individuals.

Subject age ranged from 15 to 78 years (mean \pm SD: $43.7 \pm 17.3$ years), and educational level, defined as time devoted to formal study, was 2 to 16 years $(9.9 \pm 3.7$ years). The functional and conversational fluency evaluations were not considered for this study. Testing was performed by speech pathology students trained in the administration of the BDAE and instructed to apply the tasks in a uniform procedure, while a speech therapy professor reviewed the scores obtained. Data were analyzed statistically using the MedCalc ${ }^{\circledR}$ version 6.00.114 and GraphPad InStat ${ }^{\circledR}$ version 3.05 software. Data distribution was tested by the KolmogorovSmirnov method, resulting in a non-normal distribution. Since normal distribution could not be obtained after attempts at conversion, analysis was done using the nonparametric Kruskal-Wallis and Mann-Whitney tests for the comparison of subgroup performance in the various subtests regarding gender, age and schooling. To evaluate the effect of formal education, the subjects were divided into two groups: up to 8 years of study $(\mathrm{N}=$ $19)$ and above 8 years $(N=35)$. To evaluate the age effect on performance, the subjects were divided into three age groups: up to 30 years $(\mathrm{N}=18)$, from 31 to 50 years $(\mathrm{N}=20)$ and more than 51 years $(\mathrm{N}=22)$. The age groups were compared for mean educational level by ANOVA followed by the TukeyKramer post-test. We then compared our results with those previously reported in the literature for English- and Spanish-speaking populations $(2,14)$. A value of $\mathrm{P}<0.05$ was considered statistically significant for all analyses.

\section{Results}

Table 1 shows the data obtained for our sample in the 30 BDAE subtests. The items showing greater heterogeneity were Word Discrimination, Nonverbal Agility, Visual Confrontation Naming, Animal Fluency and Serial Writing. Based on the means obtained, we present a suggested cut-off score for use in Brazil calculated from the means obtained for the subjects for the several subitems, -2 SD. Table 2 shows the distribution of the results as percentiles.

Table 3 shows the mean performance of the Portuguese-, English- and Spanish-speaking populations. Some subtests are not shown due to the absence of published results about them. In some subtests, the maximum scores in the English and Spanish versions were different from those in the Portuguese version. In the English and Spanish versions, the maximum score for the item Commands is 15 , while in Portuguese it is 11 ; in the Responsive Naming item, they are respectively 30 and 27, and in the Visual Confron- 
tation Naming subtest, 96 and 114 (the last includes the Body-Part Naming task). In Serial Writing (alphabet), there was a difference of three points related to the letters $\mathrm{K}$, $\mathrm{W}$ and $\mathrm{Y}$, which were not considered in our scoring.

The performance for male $(\mathrm{N}=21)$ and

Table 1. Performance of a Brazilian population sample in the Boston Diagnostic Aphasia Examination.

\begin{tabular}{|c|c|c|c|c|}
\hline Subtest & Mean (SD) & $95 \% \mathrm{Cl}$ & Range & $\begin{array}{l}\text { Suggested } \\
\text { cut-off score }\end{array}$ \\
\hline \multicolumn{5}{|l|}{ Oral comprehension } \\
\hline Word discrimination & $70.4(4.0)$ & $69.3-71.5$ & $48-72$ & 62 \\
\hline Body-part identification & $19.2(1.4)$ & 18.9-19.6 & $14.5-20$ & 16 \\
\hline Commands & $10.8(0.7)$ & $10.6-11$ & $7-11$ & 9 \\
\hline Complex ideational material & $10.9(1.4)$ & $10.6-11.3$ & $5-12$ & 8 \\
\hline \multicolumn{5}{|l|}{ Oral agility } \\
\hline Nonverbal agility & $9.2(2.3)$ & $8.6-9.8$ & $4-12$ & 4 \\
\hline Verbal agility & $12.6(1.9)$ & 12.1-13.1 & $7-14$ & 8 \\
\hline Automatized sequences & $7.8(0.4)$ & 7.8-8 & $6-8$ & 7 \\
\hline Recitation & $1.9(0.4)$ & $1.8-2$ & $0-2$ & 1 \\
\hline Singing & $1.9(0.4)$ & $1.8-2$ & $0-2$ & 1 \\
\hline Rhythm & $1.8(0.4)$ & $1.7-2$ & $0-2$ & 1 \\
\hline \multicolumn{5}{|l|}{ Repetition } \\
\hline Words & $9.9(0.1)$ & $9.9-10$ & $9-10$ & 9 \\
\hline High-probability phrases & $7.8(0.4)$ & 7.7-7.9 & $6-8$ & 7 \\
\hline Low-probability phrases & $7.8(0.5)$ & 7.7-7.9 & $6-8$ & 6 \\
\hline \multicolumn{5}{|l|}{ Naming } \\
\hline Responsive & $26.7(0.7)$ & $26.5-26.9$ & $24-27$ & 25 \\
\hline Visual confrontation & $112.0(4.7)$ & $110.8-113.3$ & $86-114$ & 102 \\
\hline Animal fluency & $20.3(6.1)$ & $18.5-22.1$ & $11-36$ & 8 \\
\hline \multicolumn{5}{|l|}{ Oral reading } \\
\hline Words & $29.6(1.2)$ & 29.3-30 & $22-30$ & 27 \\
\hline Sentences & $9.8(0.6)$ & $9.7-10$ & $6-10$ & 8 \\
\hline \multicolumn{5}{|l|}{ Reading comprehension } \\
\hline Symbol discrimination & $9.8(0.4)$ & $9.8-10$ & $8-10$ & 9 \\
\hline Word recognition & $7.9(0.5)$ & $7.8-8$ & $5-8$ & 7 \\
\hline Comprehension of oral spelling & 6.7 (1.9) & $6.2-7.2$ & $1-8$ & 3 \\
\hline Word-picture matching & $9.7(1.3)$ & $9.3-10$ & $1-10$ & 7 \\
\hline Sentences and paragraphs & $9.5(0.8)$ & $9.3-9.8$ & $5-10$ & 7 \\
\hline \multicolumn{5}{|l|}{ Writing } \\
\hline Writing mechanics & $4.8(0.4)$ & $4.7-5$ & $3-5$ & 4 \\
\hline Serial writing & $44.0(3.9)$ & $43-45$ & $20-45$ & 36 \\
\hline Primer-level dictation & $14.5(1.3)$ & $14.2-14.9$ & $7-15$ & 12 \\
\hline Spelling to dictation & $9.4(1.5)$ & $9-9.8$ & $2-10$ & 6 \\
\hline Written confrontation naming & $9.8(0.6)$ & $9.7-10$ & $6-10$ & 8 \\
\hline Narrative writing & $4.5(0.8)$ & $4.3-4.8$ & $2-5$ & 3 \\
\hline Sentences to dictation & $11.7(0.9)$ & $11.5-12$ & $6-12$ & 10 \\
\hline
\end{tabular}

Data are reported as means (SD). female $(\mathrm{N}=39)$ groups in the Brazilian sample did not reveal any significant differences (Table 4). There was no difference in educational level between men and women (Table 5). Analysis of performance among the three age groups revealed a significant difference in the Visual Confrontation Naming and Sentences to Dictation subtests (Table 6). There was no difference in average educational level among these groups (Table 7). Educational level (from 1 to 8 years and 9 years or more) influenced performance only in the following items: Word Discrimination and Body-Part Identification (Table 8). We will also discuss some of the answer variations found in this population, as they represent a source of knowledge about the language processes in normal subjects.

\section{Discussion}

\section{Answer variations}

Oral comprehension. Deviations noted for this item reflect knowledge from the culturally diversified universe of the individuals. In the identification of semantic fields, the largest number of deviations occurred in geometric forms (particularly spiral and cone), numbers (7 and 7000) and details of body parts (such as eyelid and thorax). However, response delay occurred randomly. In Commands, complex sequences that involved a larger number of items and actions were the most impaired. In the Complex Ideational Material (phrases) item, the low frequency of the item galosh may be the root of the errors verified in phrase 4, these being the major source of deviation in the subtest. Likewise, phrases that demand analysis of quantitative concepts provoked wrong answers. In the comprehension of texts, we observed that most individuals gave wrong answers in the subtest which involved nonliteral sense interpretation. Another text that presented difficulty was one for which interpretation was not based on common world 
knowledge (to hunt lions/lion hunting).

Oral expression. In the test of oral movements, the female group performed slower than the male group, both in nonverbal and verbal situations. The nonverbal movements constituted the major source of deviation. In the Automatized Sequences, errors were mainly in the oral presentation of the alphabet. Errors that did not compromise the meaning were noted under High-Probability Repetition. The phrase containing adverbs related to time and place, the final one being embedded (They heard him speak on the radio last night), was impaired. The HighProbability stimuli may permit a repetition without detailed analysis, inducing slight deviations. In Low-Probability Phrases, word omission and semantic changes occurred occasionally with meaning alteration. As in all other subtests, some deviations did not constitute errors, but denoted cultural deprivation (they heard = they saw - in Portuguese, these two verbs are similar in sound; Italian tie $=$ Intalian tie - denotes low culture level). In Visual Confrontation Naming the largest number of deviations occurred in number and color categories. "Cactus" incurred the most deviations among nouns and "gray" among colors. The verbs "falling" and "dripping" and the number "7000" as well as the body parts "ankle" and "shoulder" also required a longer response time. In Word Reading, "smoking", "seven hundred and twenty-one" and "brown" were read erroneously, denoting an evident effect of educational level, such as grapheme conversion which marks nasal traces and the graphic extension of the number, being related to the habit of writing checks. The writing of phrases revealed this effect more acutely. The changes introduced indicated plausible adaptations and constructions, although some were substantially distant from the target stimuli (O jiló é amargo = O Julio é amigo) . Although the sound of these phrases is similar in Portuguese, the first refers to a vegetable being bitter and the second to Julio being a friend.

Written comprehension. In the Symbol Discrimination subtest, there were deviations involving the graphemes, which represent difficulties for Portuguese language speakers. Graphemes that do not represent a strict correspondence, such as "J" (which, together with "e" and "i" leave doubts re-

Table 2. Performance of the Brazilian population sample in the Boston Diagnostic Aphasia Examination in percentiles.

\begin{tabular}{|c|c|c|c|c|c|c|c|c|c|}
\hline \multirow[t]{2}{*}{ Subtest } & \multicolumn{9}{|c|}{ Percentile } \\
\hline & 10 & 20 & 30 & 40 & 50 & 60 & 70 & 80 & 90 \\
\hline \multicolumn{10}{|l|}{ Oral comprehension } \\
\hline Word discrimination & 66.5 & 70 & 72 & & & & & & \\
\hline Body-part identification & 18 & 18.5 & 19.5 & 20 & & & & & \\
\hline Commands & 11 & 11 & & & & & & & \\
\hline Complex ideational material & 9 & 10 & 11 & & & 12 & & & \\
\hline \multicolumn{10}{|l|}{ Oral agility } \\
\hline Nonverbal agility & 6 & 7 & 8 & 8.5 & 10 & & 11 & 12 & \\
\hline Verbal agility & 10 & 11 & 12 & 13 & 14 & & & & \\
\hline Automatized sequences & 7 & 8 & & & & & & & \\
\hline Recitation & 2 & & & & & & & & \\
\hline Singing & 2 & & & & & & & & \\
\hline Rhythm & 1 & 2 & & & & & & & \\
\hline \multicolumn{10}{|l|}{ Repetition } \\
\hline Words & 10 & & & & & & & & \\
\hline High-probability phrases & 7 & 8 & & & & & & & \\
\hline Low-probability phrases & 7 & 8 & & & & & & & \\
\hline \multicolumn{10}{|l|}{ Naming } \\
\hline Responsive & 26 & 27 & & & & & & & \\
\hline Visual confrontation & 108 & 111 & 113 & 114 & & & & & \\
\hline Animal fluency & 14 & 15 & 17 & 19 & 20 & 23 & 25 & 27 & 35 \\
\hline \multicolumn{10}{|l|}{ Oral reading } \\
\hline Words & 29 & 30 & & & & & & & \\
\hline Sentences & 10 & & & & & & & & \\
\hline \multicolumn{10}{|l|}{ Reading comprehension } \\
\hline Symbol discrimination & 10 & & & & & & & & \\
\hline Word recognition & 8 & & & & & & & & \\
\hline Comprehension of oral spelling & 5 & 6 & 7 & & & 8 & & & \\
\hline Word-picture matching & 10 & & & & & & & & \\
\hline Sentences and paragraphs & 9 & & 10 & & & & & & \\
\hline \multicolumn{10}{|l|}{ Writing } \\
\hline Writing mechanics & 4 & 5 & & & & & & & \\
\hline Serial writing & 44 & 45 & & & & & & & \\
\hline Primer-level dictation & 14 & 15 & & & & & & & \\
\hline Spelling to dictation & 9 & & 10 & & & & & & \\
\hline Written confrontation naming & 10 & & & & & & & & \\
\hline Narrative writing & 4 & & 5 & & & & & & \\
\hline Sentences to dictation & 12 & & & & & & & & \\
\hline
\end{tabular}


garding choice), or those which are silent or represent a varying auditive representation such as "H". Some monosyllables such as "but" (mas) and "bar" (involving an archiphoneme with a corresponding varying

Table 3. Performances of Brazilian, North American and Colombian population samples in the Boston Diagnostic Aphasia Examination.

\begin{tabular}{|c|c|c|c|}
\hline \multirow[t]{2}{*}{ Subtest } & \multicolumn{3}{|c|}{ Language } \\
\hline & $\begin{array}{l}\text { Portuguese } \\
\qquad(\mathrm{N}=60)\end{array}$ & $\begin{array}{l}\text { English } \\
(\mathrm{N}=147)\end{array}$ & $\begin{array}{l}\text { Spanish } \\
(\mathrm{N}=156)\end{array}$ \\
\hline \multicolumn{4}{|l|}{ Oral comprehension } \\
\hline Word discrimination & $70.4(4.0)$ & $71.8(0.6)$ & $70.54(2.67)$ \\
\hline Body-part identification & $19.2(1.4)$ & $19.7(0.5)$ & $18.65(2.07)$ \\
\hline Commands* & $10.8(0.7)$ & $14.9(0.4)$ & $14.39(1.15)$ \\
\hline Complex ideational material & $10.9(1.4)$ & $11.2(1.1)$ & $9.05(1.66)$ \\
\hline \multicolumn{4}{|l|}{ Oral agility } \\
\hline Nonverbal agility & $9.2(2.3)$ & $10.4(1.5)$ & \\
\hline Verbal agility & $12.6(1.9)$ & $13.7(0.6)$ & \\
\hline Automatized sequences & $7.8(0.4)$ & $7.9(0.3)$ & \\
\hline Reciting & $1.9(0.4)$ & $1.9(0.3)$ & \\
\hline Singing & $1.9(0.4)$ & $1.8(0.5)$ & \\
\hline Rhythm & $1.8(0.4)$ & $1.8(0.5)$ & \\
\hline \multicolumn{4}{|l|}{ Repetition } \\
\hline Words & $9.9(0.1)$ & $9.9(0.3)$ & $9.98(0.11)$ \\
\hline High-probability phrases & $7.8(0.4)$ & $7.9(0.4)$ & $7.8(0.48)$ \\
\hline Low-probability phrases & $7.8(0.5)$ & $7.7(0.6)$ & $7.82(0.41)$ \\
\hline \multicolumn{4}{|l|}{ Naming } \\
\hline Responsive* & $26.7(0.7)$ & $29.9(0.5)$ & $29.32(3.64)$ \\
\hline Visual confrontation* & $112.0(4.7)$ & & $94.93(2.67)$ \\
\hline Animal fluency & $20.3(6.1)$ & $22.5(6.8)$ & $25.88(6.45)$ \\
\hline \multicolumn{4}{|l|}{ Oral reading } \\
\hline Words & $29.6(1.2)$ & & $29.87(1.22)$ \\
\hline Sentences & $9.8(0.6)$ & $9.9(0.4)$ & $9.87(0.78)$ \\
\hline \multicolumn{4}{|l|}{ Reading comprehension } \\
\hline Symbol discrimination & $9.8(0.4)$ & & $9.71(1.21)$ \\
\hline Word recognition & $7.9(0.5)$ & $7.9(0.3)$ & $7.89(0.63)$ \\
\hline Comprehension of oral spelling & $6.7(1.9)$ & $7.7(0.7)$ & $6.58(1.68)$ \\
\hline Word-picture matching & $9.7(1.3)$ & & $9.98(0.13)$ \\
\hline Sentences and paragraphs & $9.5(0.8)$ & $9.5(0.8)$ & $9.41(0.9)$ \\
\hline \multicolumn{4}{|l|}{ Writing } \\
\hline Writing mechanics & $4.8(0.4)$ & & $4.94(0.42)$ \\
\hline Serial writing* & $44.0(3.9)$ & & $46.27(5.08)$ \\
\hline Primer-level dictation & $14.5(1.3)$ & & $13.66(1.57)$ \\
\hline Spelling to dictation & $9.4(1.5)$ & $8.9(1.2)$ & $9.8(0.97)$ \\
\hline Written confrontation naming & $9.8(0.6)$ & $9.7(0.6)$ & $9.8(0.99)$ \\
\hline Narrative writing & $4.5(0.8)$ & $3.7(0.5)$ & $4.86(0.65)$ \\
\hline Sentences to dictation & $11.7(0.9)$ & $11.9(0.4)$ & 11.78 (1.36) \\
\hline
\end{tabular}

Data are reported as means (SD).

* Maximum scores are lower in the Portuguese version (see text). audiographeme) were also discriminated incorrectly. The item Oral Spelling presented great difficulty, especially for the polysyllabic word "elbow" ("cotovelo") for most of the subjects tested. The difficulty in spelling is directly related to the fact that the written Portuguese language is based on consistent rules of conversion for grapheme-phoneme. Due to this characteristic, spelling becomes less necessary.

Written expression. The deviations in the Primer-Level Dictation refer to the difficulties with the stimulus type (grapheme with a complex correspondent and numbers with a complex syntax, such as "milhar". In Spelling to Dictation, besides the above deviations, there was the absence of accents. In the Written Confrontation Naming, a discreet difficulty for numbers and colors was constant. In Dictation, deviations were noted which involved clytic pronouns ("vê-lo"/"see you"), constructions with corresponding graphics which require the insertion of a diphthong or of nasal archiphonemes and a diphthong.

\section{O verall analysis}

The first question proposed refers to the necessity of knowing the normative data for the test target population. We cannot presuppose a priori that foreign populations' scores are necessarily valid due to the previously discussed variables involved. Individuals with low instruction, totally functional in their routines, can and frequently do present performances which overlap those of patients with cerebral lesion when submitted to neuropsychological evaluation, increasing the probability of a mistaken diagnosis if the appropriate corrections are not made (1518). Furthermore, the intuitive assumption that lower efficiency is expected from the population of lower educational level conversely leads to underestimation of real pathological data. It should be noted, however, that the purpose of any neuropsychological 
test is to separate normal individuals from those with brain lesions. Some authors believe that sociocultural factors do not play such an important role in the performance of subjects with cerebral disorders, or, in other words, brain lesions "mask" any possible difference owing to their great capacity to disrupt cerebral functioning $(19,20)$.

Our impression from these preliminary data leads us to believe that the performance of the Brazilian population does not differ greatly from that of the American and Colombian samples. This is interesting when we consider that the comparison was between a Latin language and an Anglo-Saxon one and between two other Latin languages. We can then infer that the BDAE is not compromised in its applicability as a result of linguistic factors, and eventual differences between populations can probably be attributed to other demographic factors.

It is known that aging can have some detrimental impact on cognitive functions, including language; however, these alterations are in general restricted to some domains which are supported by attention, memory and executive functions and are sometimes due to sensory dysfunction (21, 22). Rosselli et al. (23) found a worsening in performance with increasing age in the subtests Word Discrimination, Body-Part Identification, Complex Ideational Material, Visual Confrontation Naming, Animal Fluency, Oral Sentence Reading Repetition of High- and Low-Probability Phrases, all subtests of Reading Comprehension, Serial Writing, Primer-Level Dictation, Written Confrontation Naming and Spelling to Dictation. Borod et al. (24) also found differences in the Commands and Automatized Sequences subtests but not in Body-Part Identification, Comprehension of Oral Spelling and Spelling to Dictation. Our data did not reproduce these results. Although we have found differences in scores for the items Visual Confrontation Naming and Sentences to Dictation when comparing subjects by
Table 4. Performance in the Boston Diagnostic Aphasia Examination by gender.

\begin{tabular}{|c|c|c|c|}
\hline \multirow[t]{2}{*}{ Subtest } & \multicolumn{2}{|c|}{ Gender } & \multirow[t]{2}{*}{ P* } \\
\hline & $\begin{array}{c}\text { Male } \\
(\mathrm{N}=21)\end{array}$ & $\begin{array}{l}\text { Female } \\
(\mathrm{N}=39)\end{array}$ & \\
\hline \multicolumn{4}{|l|}{ Oral comprehension } \\
\hline Word discrimination & $71.14(2.59)$ & $70.0(4.67)$ & 0.2766 \\
\hline Body-part identification & $19.35(1.39)$ & $19.16(1.38)$ & 0.4244 \\
\hline Commands & $10.9(0.43)$ & $10.74(0.78)$ & 0.5984 \\
\hline Complex ideational material & $11.28(1.1)$ & $10.79(1.61)$ & 0.1896 \\
\hline \multicolumn{4}{|l|}{ Oral agility } \\
\hline Nonverbal agility & $9.8(2.11)$ & $8.9(2.35)$ & 0.1935 \\
\hline Verbal agility & $12.9(1.77)$ & $12.47(2.0)$ & 0.4023 \\
\hline Automatized sequences & $7.95(0.21)$ & $7.82(0.45)$ & 0.4756 \\
\hline Recitation & $1.85(0.47)$ & $1.92(0.35)$ & 0.7799 \\
\hline Singing & $1.9(0.43)$ & $1.89(0.44)$ & 0.9867 \\
\hline Rhythm & $1.88(0.33)$ & $1.8(0.55)$ & 0.8976 \\
\hline \multicolumn{4}{|l|}{ Repetition } \\
\hline Words & $9.95(0.21)$ & $10.0(0)$ & 0.1906 \\
\hline High-probability phrases & $7.95(0.21)$ & $7.77(0.53)$ & 0.3731 \\
\hline Low-probability phrases & $8.0(0)$ & $7.71(0.61)$ & 0.7625 \\
\hline \multicolumn{4}{|l|}{ Naming } \\
\hline Responsive & $26.8(0.68)$ & $26.69(0.76)$ & 0.5907 \\
\hline Visual confrontation & $112.66(2.43)$ & $111.73(5.6)$ & 0.8651 \\
\hline Animal fluency & $21.33(7.6)$ & $19.83(5.38)$ & 0.6560 \\
\hline \multicolumn{4}{|l|}{ Oral reading } \\
\hline Words & $29.9(0.3)$ & $29.48(1.52)$ & 0.5997 \\
\hline Sentences & $9.9(0.3)$ & $9.84(0.67)$ & 0.9328 \\
\hline \multicolumn{4}{|l|}{ Reading comprehension } \\
\hline Symbol discrimination & $9.9(0.3)$ & $9.87(0.4)$ & 0.9540 \\
\hline Word recognition & $7.95(0.21)$ & $7.87(0.57)$ & 0.9734 \\
\hline Comprehension of oral spelling & $6.9(2.12)$ & $6.67(1.79)$ & 0.2871 \\
\hline Word-picture matching & $9.66(0.79)$ & $9.69(1.5)$ & 0.3830 \\
\hline Sentences and paragraphs & $9.57(0.67)$ & $9.55(0.95)$ & 0.7881 \\
\hline \multicolumn{4}{|l|}{ Writing } \\
\hline Writing mechanics & $4.83(0.38)$ & $4.8(0.49)$ & 0.9868 \\
\hline Serial writing & $44.38(3.32)$ & $44.0(4.29)$ & 0.5344 \\
\hline Primer-level dictation & $14.8(0.4)$ & $14.46(1.62)$ & 0.9612 \\
\hline Spelling to dictation & $9.2(1.54)$ & $9.56(1.46)$ & 0.2310 \\
\hline Written confrontation naming & $9.8(0.51)$ & $9.83(0.68)$ & 0.6928 \\
\hline Narrative writing & $4.6(0.82)$ & $4.54(0.75)$ & 0.6642 \\
\hline Sentences to dictation & $11.6(1.35)$ & $11.82(0.61)$ & 0.9354 \\
\hline
\end{tabular}

Data are reported as means (SD). *Mann-Whitney test.

Table 5. Correlation between educational level and gender.

\begin{tabular}{lccc}
\hline & \multicolumn{2}{c}{ Gender } & P* \\
\cline { 2 - 3 } & Male $(\mathrm{N}=21)$ & Female $(\mathrm{N}=39)$ & \\
\hline Schooling (years) & $10.38(4.17)$ & $9.66(3.5)$ & 0.1903 \\
\hline Data are reported as means (SD). * Unpaired t-test. & &
\end{tabular}


Table 6 - Performance in the Boston Diagnostic Aphasia Examination by age group.

\begin{tabular}{|c|c|c|c|c|}
\hline \multirow[t]{2}{*}{ Subtest } & \multicolumn{3}{|c|}{ Age groups (years) } & \multirow[t]{2}{*}{ P* } \\
\hline & $\begin{array}{c}A(15-30) \\
(N=18)\end{array}$ & $\begin{array}{l}B(31-50) \\
(N=20)\end{array}$ & $\begin{array}{l}C(51-78) \\
(\mathrm{N}=22)\end{array}$ & \\
\hline \multicolumn{5}{|l|}{ Oral comprehension } \\
\hline Word discrimination & 71.41 (1.66) & $70.0(4.0)$ & $69.95(5.27)$ & 0.3343 \\
\hline Body-part identification & $19.11(1.61)$ & $19.25(1.46)$ & $19.31(1.14)$ & 0.9401 \\
\hline Commands & $10.88(0.47)$ & $10.9(0.3)$ & $10.63(1.0)$ & 0.6713 \\
\hline Complex ideational material & $11.16(1.34)$ & $10.73(1.79)$ & $11.0(1.27)$ & 0.6936 \\
\hline \multicolumn{5}{|l|}{ Oral agility } \\
\hline Nonverbal agility & $9.55(2.0)$ & $8.95(2.18)$ & $9.19(2.67)$ & 0.7979 \\
\hline Verbal agility & $13.0(1.3)$ & $13.0(1.49)$ & $11.9(2.5)$ & 0.3121 \\
\hline Automatized sequences & $7.88(0.47)$ & $7.85(0.36)$ & $7.86(0.35)$ & 0.6792 \\
\hline Recitation & $1.76(0.66)$ & $1.9(0.3)$ & $2.0(0)$ & 0.2733 \\
\hline Singing & $2.0(0)$ & $1.8(0.61)$ & $1.9(0.42)$ & 0.3723 \\
\hline Rhythm & $1.81(0.4)$ & $1.8(0.53)$ & $1.84(0.5)$ & 0.8679 \\
\hline \multicolumn{5}{|l|}{ Repetition } \\
\hline Words & $10.0(0)$ & $10.0(0)$ & $9.95(0.21)$ & 0.4216 \\
\hline High-probability phrases & $7.88(0.47)$ & $7.75(0.55)$ & $7.86(0.35)$ & 0.4607 \\
\hline Low-probability phrases & $7.94(0.23)$ & $7.65(0.67)$ & $7.85(0.49)$ & 0.1837 \\
\hline \multicolumn{5}{|l|}{ Naming } \\
\hline Responsive & $27.0(0)$ & $26.6(0.82)$ & $26.63(0.9)$ & 0.0936 \\
\hline Visual confrontation & $113.55(1.46)$ & $111.1(4.98)$ & $111.68(5.96)$ & $0.0343 * *$ \\
\hline Animal fluency & $20.46(6.18)$ & $21.33(4.32)$ & $19.25(7.6)$ & 0.3897 \\
\hline \multicolumn{5}{|l|}{ Oral reading } \\
\hline Words & $29.75(0.77)$ & $29.72(0.57)$ & $29.47(1.83)$ & 0.6269 \\
\hline Sentences & $9.94(0.23)$ & $9.89(0.31)$ & $9.77(0.87)$ & 0.8559 \\
\hline \multicolumn{5}{|l|}{ Reading comprehension } \\
\hline Symbol discrimination & $10.0(0)$ & $9.75(0.55)$ & $9.9(0.29)$ & 0.1197 \\
\hline Word recognition & $7.94(0.23)$ & $7.85(0.67)$ & $7.9(0.42)$ & 0.9922 \\
\hline Comprehension of oral spelling & 7.27 (1.7) & $6.5(1.82)$ & $6.55(2.14)$ & 0.1377 \\
\hline Word-picture matching & $9.77(0.73)$ & $9.45(2.0)$ & $9.81(0.66)$ & 0.9772 \\
\hline Sentences and paragraphs & $9.61(0.6)$ & $9.57(0.76)$ & $9.5(1.1)$ & 0.9744 \\
\hline \multicolumn{5}{|l|}{ Writing } \\
\hline Writing mechanics & $4.85(0.37)$ & $4.87(0.5)$ & $4.73(0.45)$ & 0.3611 \\
\hline Serial writing & $45.0(0.53)$ & $44.6(2.3)$ & $43.0(6)$ & 0.1577 \\
\hline Primer-level dictation & $14.83(0.51)$ & $14.6(1.35)$ & $14.36(1.73)$ & 0.3939 \\
\hline Spelling to dictation & $9.77(0.42)$ & $9.68(0.75)$ & $8.9(2.31)$ & 0.8214 \\
\hline Written confrontation naming & $10.0(0)$ & $9.89(0.31)$ & $9.63(0.95)$ & 0.1766 \\
\hline Narrative writing & $4.6(0.63)$ & $4.55(0.85)$ & $4.55(0.82)$ & 0.9796 \\
\hline Sentences to dictation & $12.0(0)$ & $12.0(0)$ & $11.36(1.46)$ & $0.0157^{+}$ \\
\hline
\end{tabular}

Data are reported as means (SD). *Kruskal-Wallis with Müller-Dunn post-test. ** Group difference: A-B. ${ }^{+}$Group difference: A-C and B-C.

Table 7. Correlation between educational level and age group.

\begin{tabular}{lcccc}
\hline & \multicolumn{3}{c}{ Age (years) } & P* \\
\cline { 2 - 5 } & $15-30(\mathrm{~N}=18)$ & $31-50(\mathrm{~N}=20)$ & $51-78(\mathrm{~N}=22)$ & \\
\hline Schooling (years) & $11.14(1.9)$ & $10.42(4.1)$ & $8.52(3.9)$ & 0.0894
\end{tabular}

Data are reported as means (SD). *ANOVA with Tukey post-test. age, a case-by-case analysis of the worst scores does not allow us to exclude a coparticipation of the low educational level factor. These two variables were superimposed in five of the eight worst scores for Visual Confrontation Naming and in two of the four worst scores for Sentences to Dictation. This issue could be properly reconsidered in a study on a larger number of subjects. Correlation between a decrease in spontaneous language production and aging with superimposed effects of educational level by sex was reported by Ardila and Rosselli (25) in a study based on the oral description of "The Cookie Theft" picture.

Gender differences are controversial in the literature $(26,27)$. When positive, they show a better performance of women in some subtests, especially in naming, reading and writing tasks. Our results did not reproduce these findings.

Illiteracy can compromise different aspects of language, such as meta-phonological analysis, oral comprehension, short-term memory and grammatical judgment (28), leading to a poorer performance in language and other neuropsychological batteries (29, 30). There are few consistent studies on low educational level such as those commonly seen in public health assistance services in Brazil. Correlation between schooling and language abilities was found in studies involving the BDAE in the English and Spanish versions. In these studies the authors verified that scores were impaired especially among subjects with less than 9 years of education. In the Colombian population, academic achievement affected the scores in the items Animal Fluency, Word Reading, Oral Sentence Reading, Symbol Discrimination, Oral Spelling, Word-Picture Matching, Serial Writing, Written Confrontation Naming, Spelling to Dictation and Sentences to Dictation $(24,26)$.

We identified an influence of number of years of formal study and performance on two items: Word Discrimination and Body- 
Part Identification. The attempt to compare individuals by dividing them into groups with less and more than 8 years of formal study (as done in the American and Colombian studies reported) failed to reproduce their findings. This observation indicates that such a division was not able to show the critical number of years of formal education that exerts the greatest impact on subject performance. This, in turn, points to the necessity of studying in greater depth the group with less than 8 years of education. This was not done in the present study due to the small sample size for this category. We should also note that this correlation between educational level and performance in neuropsychological tests does not occur linearly: the two years from 0 to literacy obviously have much more impact than the interval from 6 to 8 years and so on, which calls for caution in the interpretation of data relative to this variable.

The principal purpose of the BDAE is to detect aphasia. We understand that its application in Portuguese will reach the sensitivity already demonstrated in English only when precise indicators of our sociocultural reality are considered, an objective that was partially reached in the present study. The present adaptation respects the authors' aims and proposed coherence. It allows the scanning of several aspects related to comprehension and production processing, quantification of the intensity of impairment and a qualitative analysis of functional interactions among different language system components. The limits of its application are those inherent to aphasia diagnostic tests: the answers obtained represent only a sample of the relevant aspects of subject performance. Using this test, it is not possible to assess the language disorder in its entirety and the preserved language domain. When the purpose of assessment is counseling the patient's family and specifying a therapeutic plan, it is necessary to apply other tools to obtain complementary information about the un- derlying cognitive deficits and communication competence. The scores obtained should not be analyzed separately, but within the set of tasks, in order to appreciate the nature of the deficit and its impact on the language disorder. Variations for normal subjects that may represent attention lapses or cultural

Table 8. Performance in the Boston Diagnostic Aphasia Examination by educational level.

\begin{tabular}{|c|c|c|c|}
\hline \multirow[t]{2}{*}{ Subtest } & \multicolumn{2}{|c|}{ Schooling (years) } & \multirow[t]{2}{*}{$\mathrm{P}^{*}$} \\
\hline & $1-8(\mathrm{~N}=19)$ & $>9(\mathrm{~N}=35)$ & \\
\hline \multicolumn{4}{|l|}{ Oral comprehension } \\
\hline Word discrimination & $68.32(6.21)$ & 71.44 (1.92) & 0.0108 \\
\hline Body-part identification & $18.63(1.65)$ & $19.61(0.94)$ & 0.0201 \\
\hline Commands & $10.57(1.07)$ & $10.88(0.4)$ & 0.6041 \\
\hline Complex ideational material & $10.31(1.91)$ & $11.26(1.0)$ & 0.0623 \\
\hline \multicolumn{4}{|l|}{ Oral agility } \\
\hline Nonverbal agility & $8.57(2.52)$ & $9.42(2.17)$ & 0.2038 \\
\hline Verbal agility & $11.78(2.5)$ & $13.0(1.4)$ & 0.1045 \\
\hline Automatized sequences & $7.78(0.41)$ & $7.91(0.37)$ & 0.3518 \\
\hline Recitation & $1.94(0.22)$ & $1.85(0.5)$ & 0.8121 \\
\hline Singing & $1.68(0.74)$ & $2.0(0)$ & 0.3416 \\
\hline Rhythm & $1.66(0.72)$ & $1.92(0.27)$ & 0.4647 \\
\hline \multicolumn{4}{|l|}{ Repetition } \\
\hline Words & $9.94(0.23)$ & $10.0(0)$ & 0.7513 \\
\hline High-probability phrases & $7.73(0.56)$ & $7.85(0.43)$ & 0.5481 \\
\hline Low-probability phrases & $7.72(0.66)$ & $7.82(0.45)$ & 0.8356 \\
\hline \multicolumn{4}{|l|}{ Naming } \\
\hline Responsive & $26.52(0.84)$ & $26.8(0.72)$ & 0.1716 \\
\hline Visual confrontation & $109.94(7.47)$ & $112.94(2.14)$ & 0.0653 \\
\hline Animal fluency & $17.78(5.71)$ & $20.86(5.71)$ & 0.0567 \\
\hline \multicolumn{4}{|l|}{ Oral reading } \\
\hline Words & $29.18(2.0)$ & $29.78(0.65)$ & 0.4336 \\
\hline Sentences & $9.66(0.97)$ & $9.94(0.23)$ & 0.4844 \\
\hline \multicolumn{4}{|l|}{ Reading comprehension } \\
\hline Symbol discrimination & $9.73(0.56)$ & $9.94(0.23)$ & 0.3227 \\
\hline Word recognition & $7.73(0.8)$ & $7.97(0.17)$ & 0.6132 \\
\hline Comprehension of oral spelling & $5.7(2.56)$ & $7.0(1.44)$ & 0.0745 \\
\hline Word-picture matching & $9.26(2.15)$ & $9.85(0.55)$ & 0.6108 \\
\hline Sentences and paragraphs & $9.22(1.21)$ & $9.65(0.64)$ & 0.1604 \\
\hline \multicolumn{4}{|l|}{ Writing } \\
\hline Writing mechanics & $4.66(0.61)$ & $5.0(0)$ & 0.1931 \\
\hline Serial writing & $42.15(6.68)$ & $44.97(0.17)$ & 0.1248 \\
\hline Primer-level dictation & $14.0(2.19)$ & $14.82(0.51)$ & 0.2081 \\
\hline Spelling to dictation & $8.52(2.47)$ & $9.82(0.38)$ & 0.1658 \\
\hline Written confrontation naming & $9.55(1.0)$ & $9.94(0.23)$ & 0.2942 \\
\hline Narrative writing & $4.29(1.1)$ & $4.7(0.52)$ & 0.3924 \\
\hline Sentences to dictation & $11.29(1.61)$ & $12.0(0)$ & 0.1765 \\
\hline
\end{tabular}

Data are reported as means (SD). * Mann-Whitney test. 
variations serve as guidelines for the qualitative analysis of errors in aphasic patients. To guarantee uniform scoring criteria for the answers, instructions must be supplied to the professionals administering the test, so that they will accept the lexicon (that allows variations in the naming tasks) and disregard graphemes that are not contained in our alphabet. We believe that the preliminary findings for this sample can contribute as a reference and be used as guidelines to determine both quantitative and qualitative errors. The standard deviations obtained in the tasks indicate the possibility of a reliable use of scores from the adapted version of the BDAE in the State of São Paulo, without distortion, but normative data obtained for a larger population sample from other regions of Brazil and especially of low educational level would provide more reliable normal-pathologic boundaries.

\section{Acknowledgments}

The authors would like to thank the students of the Speech Pathology Graduation Course, University of São Paulo, for their important participation in collecting the data.

\section{References}

1. Goodglass H \& Kaplan E (1972). The Assessment of Aphasia and Related Disorders. Lea \& Febiger, Philadelphia, PA, USA.

2. Goodglass H \& Kaplan E (1983). The Assessment of Aphasia and Related Disorders. 2nd edn. Lea \& Febiger, Philadelphia, PA, USA.

3. Goodglass H \& Kaplan E (1979). Evaluación de la Afasia y de Trastornos Similares (The Assessment of Aphasia and Related Disorders). Editorial Médica Panamericana, Buenos Aires, Argentina.

4. Goodglass H \& Kaplan E (1986). Evaluación de la Afasia y de Trastomos Similares (The Assessment of Aphasia and Related Disorders). 2nd edn. Editorial Médica Panamericana, Madrid, Spain.

5. Goodglass H, Klein B, Carey PW \& J ones KJ (1972). Specific semantic word categories in aphasia. In: Samo MT (Editor), Aphasia: Selected Readings. AppletonCentury-Crofts, New York, NY, USA.

6. Caramazza A, Hillis $A E$, Rapp $B C \&$ Romani C (1990). The multiple semantics hypothesis: multiple confusions? Cognitive Neuropsychology, 7: 161-189.

7. Warrington E \& Shallice T (1984). Category-specific semantic impairments. Brain, 107: 829-854.

8. Gainotti G (1998). Category-specific disorders for nouns and verbs: a very old and very new problem. In: Stemmer B \& Whitaker $\mathrm{H}$ (Editors), Handbook of Neurolinguistics. Academic Press, San Diego, CA, USA.

9. Damasio H, Grabowski TJ, Tranel D,
Hichwa RD \& Damasio A (1996). A neural basis for lexical retrieval. Nature, 380: 499505.

10. Murtha $S$, Cherkow $H$, Beauregard $M \&$ Evans A (1999). The neural substrate of picture naming. J ournal of Cognitive Neuroscience, 11: 399-423.

11. Federmeier KD, Segal J B, Lombrozo $T$ \& Kutas M (2000). Brain responses to nouns, verbs and class-ambiguous words in context. Brain, 123: 2552-2556.

12. Mummery $\mathrm{CJ}$, Patterson $\mathrm{K}$, Hodges J R \& Price CJ (1998). Functional neuroanatomy of the semantic system: divisible by what? J ournal of Cognitive Neuroscience, 10: 766-777.

13. Willmes K (1998). Methodological and statistical considerations in cognitive neurolinguistics. In: Stemmer B \& Whitaker $\mathrm{H}$ (Editors), Handbook of Neurolinguistics. Academic Press, San Diego, CA, USA.

14. Pineda DA, Mejia SE, Rosselli M, Ardila A, Romero G \& Pérez C (1998). Variabilidad en la prueba de Boston para el diagnóstico de las afasias en adultos laboralmente activos. Revista de Neurología, 26: 962970.

15. Strub RL \& Black FW (1993). Higher cognitive functions. In: The Mental Status Examination in Neurology. 3rd edn. F.A. Davis Company, Philadelphia, PA, USA.

16. Rosselli M \& Ardila A (1990). Effects of age, education and gender on the ReyOsterrieth complex figure. Clinical Neuropsychology, 5: 370-376.

17. Ostrosky F, Canseco E, Quintanar L, Navaro E, Meneses S \& Ardila A (1985).
Sociocultural effects in neuropsychological assessment. International J ournal of Neuroscience, 27: 53-66.

18. Stern Y, Andrews H \& Pitman J (1992). Diagnosis of dementia in a heterogeneous population: Development of a neuropsychological paradigm-based diagnosis of dementia and quantified correction for the effects of education. Archives of Neurology, 40: 8-14.

19. Reitan RM \& Wolfson D (1995). Influence of age and education on neuropsychological test results. Clinical Neuropsychology, 9: 151-158.

20. Heaton RK, Grant I \& Matthews CG (1986). Differences in neuropsychological test performance associated with age, education, and sex. In: Grant I \& Adams KM (Editors), Neuropsychological Assessment of Neuropsychiatric Disorders. Oxford University Press, New York, NY, USA.

21. Mittenberg W, Sidenberg M, O'Leary DS $\&$ DiGiulio DV (1989). Changes in cerebral functioning associated with normal aging. J ournal of Clinical and Experimental Neuropsychology, 11: 918-932.

22. Ryan E (1995). Normal aging and language. In: Lubinski R (Editor), Dementia and Communication. Singular-Publishing, San Diego, CA, USA.

23. Rosselli M, Ardila A, Florez A \& Castro $C$ (1990). Normative data on the Boston Diagnostic Aphasia Examination in a Spanish-speaking population. J ournal of Clinical and Experimental Neuropsychology, 12: 313-322.

24. Borod J C, Goodglass H \& Kaplan E (1980). 
Normative data on the Boston Diagnostic Aphasia Examination and the Boston Naming Test. J ournal of Clinical Neuropsychology, 2: 209-215.

25. Ardila A \& Rosselli M (1996). Spontaneous language production and aging: sex and educational effects. International J ournal of Neuroscience, 87: 71-78.

26. Pineda DA, Rosselli M, Ardila A, Mejia SE, Romero MG \& Pérez C (2000). The Boston Diagnostic Aphasia Examination -
Spanish Version: Influence of demographic variables. J ournal of the International Neuropsychological Society, 6: 802814.

27. Kremin H, Deloche G, Metz-Lutz M-N, Hannequin $D$, Dordain $M$, Perrier $D$, Cardebat D, Ferrandi I, Larroque C, Naud E, Pichard B \& Bunel G (1991). The effects of age, educational background and sex on confrontation naming in normals; principals for testing naming ability.
Aphasiology, 5: 579-582.

28. Matute E (1998). Neuropsicologia del analfabetismo. Neuropsychologia Latina, 4: 38-47.

29. Rosselli M (1993). Neuropsychology of illiteracy. Behavioural Neurology, 6: 107112.

30. Rosselli M, Ardila A \& Rosas P (1990). Neuropsychological assessment in illiterates. II. Language and praxic abilities. Brain and Cognition, 12: 281-296. 